\title{
Fine distribution of CLOCK protein in hepatocytes of hibernating dormice
}

\author{
M. Malatesta, ${ }^{*}$ B. Baldelli, ${ }^{*}$ L. Rossi, ${ }^{\circ}$ S. Serafini, ${ }^{\circ}$ G. Gazzanelli* \\ *Istituto di Istologia ed Analisi di Laboratorio; ' Istituto di Chimica Biologica "G. Fornaini", \\ University of Urbino, Urbino, Italy
}

(C)2003, European Journal of Histochemistry

CLOCK protein is a member of the bHLH-PAS family of transcription factors, it is expressed in several tissues including the liver and is essential for normal circadian rhythms. In this study we investigate the distribution of CLOCK protein in hepatocytes of euthermic and hibernating edible dormice Glis glis as well as in hepatocytes taken from the hibernating animals submitted in vitro to experimental conditions mimicking the arousal process. Our results demonstrate that CLOCK protein is expressed in all animals and is mostly located in the nucleus, in particular, on perichromatin fibrils and nucleoli. During deep hibernation CLOCK protein becomes more abundant but an intracellular redistribution occurs: the protein significantly decreases in all cellular compartments, but it accumulates in the amorphous bodies. These nuclear bodies, typical of the hibernating state, probably represent storage sites for CLOCK protein to be quickly used upon arousal. Accordingly, in hepatocytes submitted to in vitro conditions mimicking arousal CLOCK protein levels rapidly reach the euthermic values, while amorphous bodies disappear.

Key words: cell nucleus, circadian rhythm, clock protein, hibernation, immunocytochemistry, liver.

Correspondence: Dr. Manuela Malatesta, Istituto di Istologia ed Analisi di Laboratorio, University of Urbino, via Zeppi s.n., 61029 Urbino, Italy. Phone: international +39.0722. 320168. Fax: international +39.0722.322370. E-mail: malatesta@uniurb.it

Paper accepted on April 11, 2003

European Journal of Histochemistry 2003; vol. 47 issue 3 [Jul-Sept]: 233-240
Hibernating animals undergo drastic modifications of their activity during the seasonal cycle: in winter, when the habitat conditions become unfavourable, they enter torpor bouts drastically reducing body temperature and energy needs; upon arousal the thermogenic processes rapidly restore euthermic metabolic and physiological functions (Lyman et al., 1982). Therefore, in hibernating animals physiological and metabolic variables follow a circannual rhythm, which has been extensively studied in many species (review in Körtner and Geiser, 2000). By contrast, little is known about their circadian rhythms; in addition, the available data are often discordant, probably due to the extreme variability of the hibernation strategies employed by different species and/or to different experimental designs.

Recently, a new member of the bHLH-PAS family of transcription factors, named CLOCK, has been identified in the mouse and demonstrated to be essential for normal circadian rhythms (Vitaterna et al., 1994). In mammals, the primary circadian pacemaker resides in the hypothalamic suprachiasmatic nuclei; however, extraneural tissues contain their own circadian oscillators (e.g. King et al., 1997; Zylka et al., 1998; Sakamoto et al., 1998). Cells and tissues are able to maintain their own circadian clock even when submitted to in vitro conditions (Balsalobre et al., 1998; Whitmore et al., 1998). The basic mechanism of the circadian clock relies on interlocking positive and negative transcriptional/translational feedback loops (review in Reppert and Weaver, 2001). The internal timekeeping system in mammals includes several genes besides clock, namely, period (per) 1, 2 and 3, cryptochrome (cry) 1 and 2 and bmall. The proteins CLOCK and BMALI form a complex that activates per and cry genes transcription; as the PER and CRY proteins are translated, they enter the nucleus where CRY acts as negative regulator interacting with CLOCK and/or BMAL1 to inhibit transcrip- 
tion, while PER2 contributes to the rhythmic transcription of bmall. Increased availability of BMALI presumably promotes CLOCK-BMALI heterodimerization needed to restart the per and cry transcription. The positive-feedback loop thereby augments regulation of the negative-feedback loop, perpetuating the clock cycle that, by secondary pathways, elicits cyclic events in other cellular targets.

In the present study, we investigate, by means of biochemical and immunoelectron microscopic techniques, the distribution of CLOCK protein in hepatocytes of euthermic and hibernating edible dormice, with particular attention to the nuclear structural constituents involved in transcription and splicing previously demonstrated to modify during hibernation (Zancanaro et al., 1993; Malatesta et al., 1994, 1995, 1999, 2000). Moreover, we analyse CLOCK protein distribution in hepatocytes submitted to in vitro experimental conditions mimicking the arousal process (Malatesta et al., 2001) in order to verify a possible role of this protein in the transitions involved in hibernation-arousal.

\section{Materials and Methods}

\section{Animals and tissue processing}

Seven male adult individuals of the edible dormouse Glis glis were used in this study. Wild animals were trapped in central Italy upon permission from local authorities and maintained in an external animal house provided with food and bedding material; under such conditions they spontaneously began to hibernate in December and awoke in March. Four animals were sacrificed in winter (three in deep hibernation, after at least six days of continuous torpor and one in early hibernation, 36 hours after a periodic arousal) and three during the euthermic summer period. Dormant animals were taken from the cage and immediately killed by cervical dislocation, whereas euthermic animals were anaesthetised with ether and then sacrificed. All animals were killed at the same time, i.e. around 10:00 am. Samples of liver were fixed by immersion in $4 \%$ paraformaldehyde in $0.1 \mathrm{M}$ Sörensen phosphate buffer at $4^{\circ} \mathrm{C}$ for $2 \mathrm{~h}$. After blocking free aldehydes with $0.5 \mathrm{M} \mathrm{NH}{ }_{4} \mathrm{Cl}$ in PBS at $4^{\circ} \mathrm{C}$ for 45 min, the specimens were dehydrated in ethanol and embedded in LRWhite resin. Some liver samples were immersed in liquid nitrogen and then stored at $-20^{\circ} \mathrm{C}$ for biochemical analyses.

\section{Electrophoresis and Western blotting}

Dormouse liver extracts were prepared by homogenizing the minced tissue $(0.05 \mathrm{~g})$ at $4^{\circ} \mathrm{C}$ in a glass potter with a Teflon pestle in $300 \mu$ l of extraction solution $(20 \mathrm{mM}$ Hepes/ $\mathrm{KOH}$ pH 7.5, $100 \mathrm{mM}$ $\mathrm{KCl}, 5 \mathrm{mM}$ dithiothreitol, $5 \%$ glycerol, $2.5 \mathrm{mM}$ EDTA, $2.5 \mathrm{mM}$ NaF, 0.1\% Triton X-100, $1 \mathrm{mM}$ PMSF, $4 \mathrm{mM}$ AEBSF, $10 \mu \mathrm{g} / \mathrm{mL}$ leupeptin, 10 $\mu \mathrm{g} / \mathrm{mL}$ pepstatin). Homogenates were centrifuged for 10 min at $12000 \mathrm{~g}$ at $4^{\circ} \mathrm{C}$ and the supernatants used for protein determination. Protein concentration was determined by the method of Bradford (1976) using BSA as standard protein. The supernatant fractions were diluted $1: 1$ in extraction solution, then an equal volume of $2 x$ SDS sample buffer (with 2\% v/v mercaptoethanol) was added and the mixture boiled for five minutes. Equal amounts of total protein $(30 \mu \mathrm{g}$ total at $1 \mu \mathrm{g} / \mu \mathrm{L})$ from the supernatant fractions were resolved by SDS/PAGE on $8 \%$ polyacrylamide gel and transferred to nitrocellulose membrane (Hybond-C extra, Amersham Life Science) (Laemmli, 1970; Towbin et al., 1979). The nitrocellulose membranes were then incubated with an affinity-purified polyclonal rabbit anti-mouse CLOCK antibody diluted 1:2000 (Alpha Diagnostic International, Inc.). Goat antirabbit IgG horseradish peroxidase conjugate (BioRad) was used at a 1:5000 dilution as a secondary antibody and specific protein bands were visualised by the ECL (enhanced chemiluminescence) detection kit as recommended by the manufacturer (Amersham Life Sciences). Densitometric analysis of the bands was performed by using Chemi Doc apparatus (Bio-Rad) and quantified by Quantity One software.

\section{In vitro experimental conditions}

Liver samples from deeply hibernating dormice were put in a cold culture medium, cut in small fragments and incubated as previously described (Malatesta et al., 2001): 1) at $4^{\circ} \mathrm{C}$, to preserve the hypothermic state of the hibernating organism, for $5,30,60$ min and $24 \mathrm{~h} ; 2$ ) at $37^{\circ} \mathrm{C}$, to simulate the drastic increase in body temperature occurring during arousal, for $5 \mathrm{~min} ; 3)$ at $37^{\circ} \mathrm{C}$, in a culture medium containing $10^{-5} \mathrm{M}$ delta opioid D-Ala2-DLeu5 enkephalin (DADLE, Sigma), which mimics the activity of the hibernation induction trigger in hibernators (Oeltgen et al., 1988), for 5, 30 and 60 min. As previously demonstrated (Malatesta et al., 2001), DADLE administered at $37^{\circ} \mathrm{C}$ is able to 
slow down the arousal process, while the preservation of the cell features typical of deep hibernation is probably possible only by a concomitant hypothermia. After incubation, all liver samples were fixed and processed for electron microscopy as described above.

\section{Immunoelectron microscopy}

Ultrathin sections were placed on nickel grids coated with a Formvar-carbon layer and then processed for immunocytochemistry using an antimouse CLOCK antibody (Alpha Diagnostic International, Inc.). Sections were floated for 3 min on normal goat serum (NGS) diluted $1: 100$ in PBS and then incubated for $17 \mathrm{~h}$ at $4^{\circ} \mathrm{C}$ with the primary antibody diluted 1:5 with PBS containing $0.1 \%$ bovine serum albumin (Fluka) and $0.05 \%$ Tween 20. After rinsing, sections were floated on NGS, and then reacted for $30 \mathrm{~min}$ at room temperature with the secondary $12 \mathrm{~nm}$-gold-conjugated antibody (Jackson ImmunoResearch) diluted 1:10 in PBS. Finally, the sections were rinsed and airdried. As controls, some grids were treated with the incubation mixture without the primary antibody, and then processed as described above. In order to clearly identify the nuclear structural constituents containing RNPs, all sections were stained with the EDTA method (Bernhard, 1969). The specimens were observed in a Zeiss EM 902 electron microscope operating at $80 \mathrm{kV}$.

\section{Quantitative evaluation of immunolabelling and statistical analysis}

In order to assess the presence of the CLOCK protein quantitatively, the labelling density was evaluated over some cellular compartments: condensed chromatin, interchromatin granules (IG), amorphous bodies (ABs), coiled bodies ( $C B s)$, fibro-granular material ( $F G M)$, interchromatin space devoid of IG and of nuclear bodies typical of hibernation (therefore a nucleoplasmic region particularly rich in perichromatin fibrils, PF), nucleoli and cytoplasm. The surface area of each compartment was measured on 15 randomly selected electron micrographs (x20000) from each animal or experimental condition by using a computerised image analysis system (Image Pro-Plus for Windows 95). For background evaluation resin outside the tissue was considered. The gold grains present over each compartment were counted and the labelling density was expressed as the number of

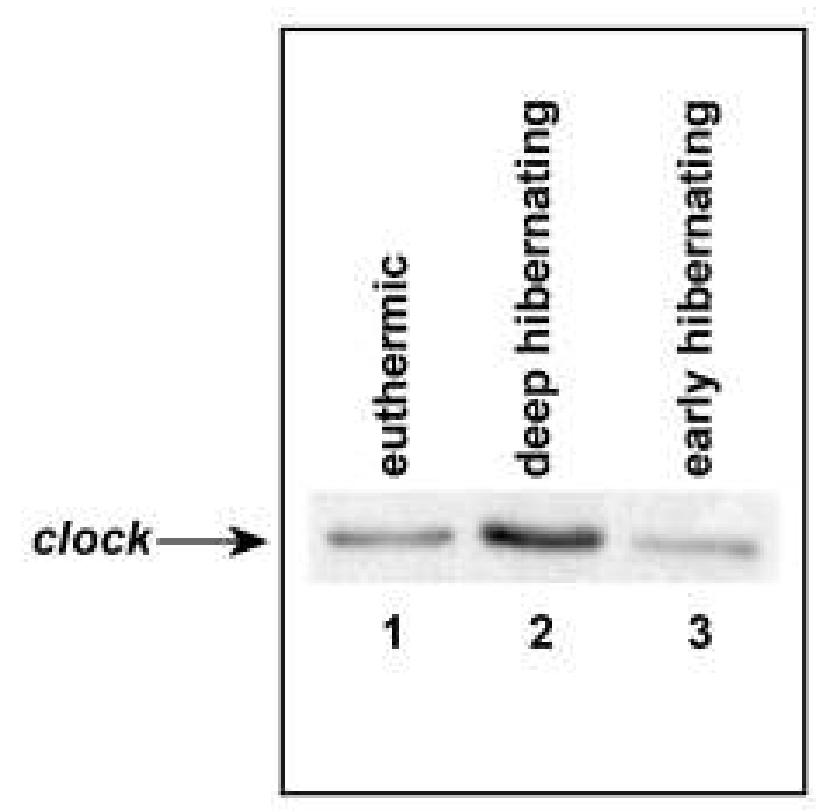

Figure 1. Immunoblot analysis of total liver extracts from euthermic (line 1), deep (line 2) and early (line 3) hibernating dormice. Thirty micrograms of protein was electrophoresed on a $8 \%(w / v)$ polyacrylamide gel and electro blotted onto nitrocellulose membrane and probed with an affinity-purified polyclonal rabbit anti-mouse CLOCK antibody. Enhanced chemiluminescence was used as the detection system.

gold grains per square micrometer.

The data for each variable were then pooled according to the experimental groups and the mean \pm standard error of the mean (SE) values calculated. Statistical analysis was performed by the Kruskal-Wallis one-way ANOVA test and relative procedures of multiple comparison. Statistical significance was set at $p \leq 0.05$.

\section{Results}

\section{CLOCK protein in liver homogenates}

CLOCK protein expression in livers of euthermic and hibernating dormice was evaluated by immunoblot analysis using an anti-mouse CLOCK antibody. The results (Figure 1 ) show the ability of this antibody to recognise the dormouse CLOCK protein. Moreover, our results indicate that hepatic tissues of deeply hibernating dormice express CLOCK protein at higher levels than euthermic and early hibernating ones ( $26 \%$ and $12.5 \%$ compared to deeply hibernating dormice, respectively).

\section{Immunoelectron microscopy}

Electron microscopic examination of immunolabeled hepatocytes revealed that the general intra- 


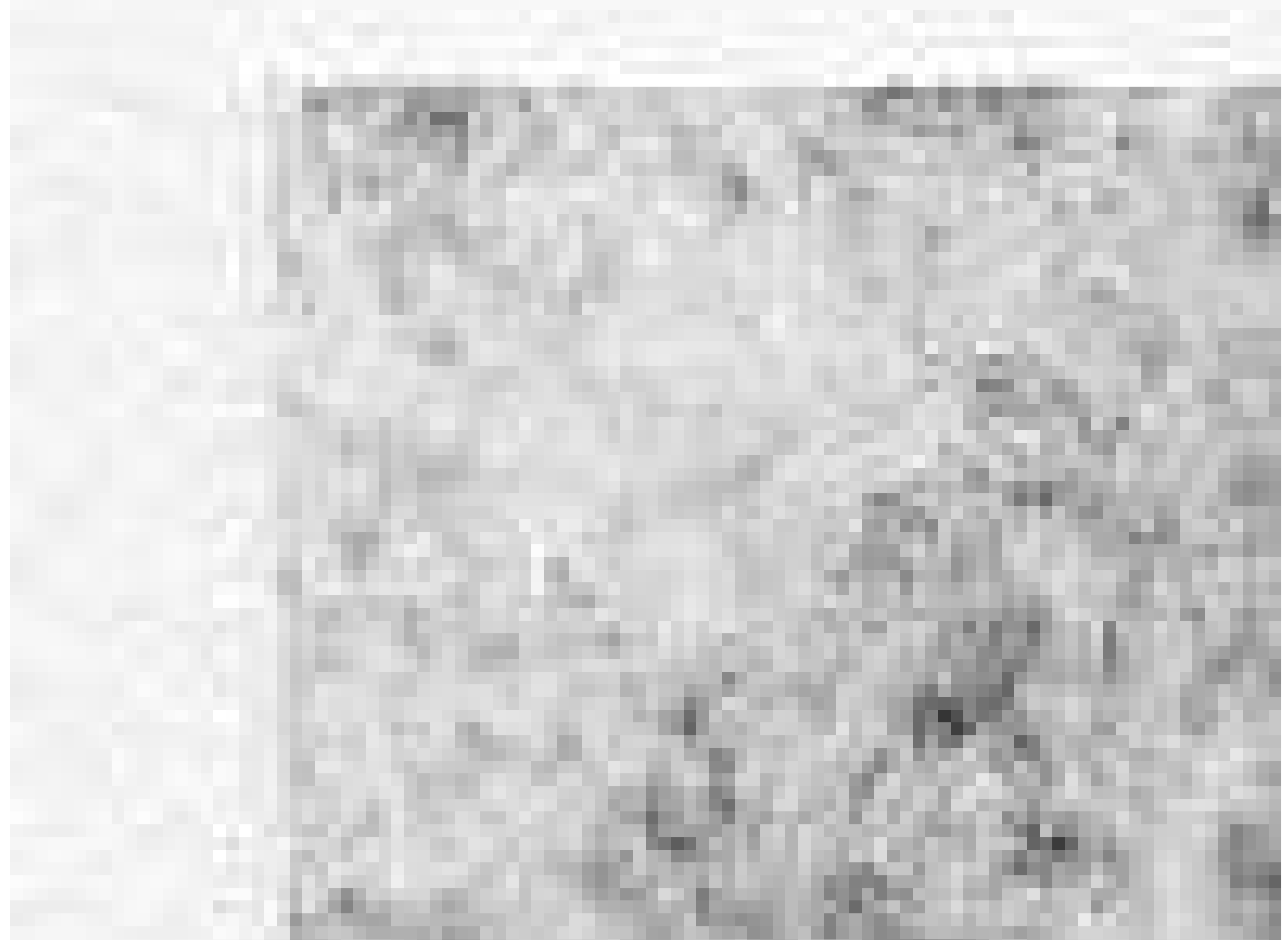

Figure 2. Hepatocytes from euthermic (a-b) and hibernating (c-e) dormice; immunolabelling with anti-CLOCK antibody. In the cytoplasm CLOCK protein labelling is associated with ribosomes (inset, arrowheads), while in the nucleus it mainly occurs on perichromatin fibrils (thin arrows) and on the dense fibrillar component (thick arrows) in the nucleolus (NU). The condensed chromatin (C) shows only a weak labelling, while interchromatin granules (IG) and perichromatin granules (small arrows) are devoid of gold grains. During deep hibernation, CLOCK protein accumulates in the amorphous bodies (arrow), whereas coiled bodies (open arrow) and fibro-granular material (asterisks) do not show any signal. Bars $0.5 \mu \mathrm{m}$; inset bar $0.25 \mu \mathrm{m}$.

cellular distribution of CLOCK protein was similar in all samples. In detail, the labelling appeared concentrated in the nucleus, where it was mostly associated with the PF, while the condensed chromatin showed a weak signal mainly located near the perichromatin region (Figure $2 \mathrm{a}$ ). The nucleolus displayed a significant labelling especially on the dense fibrillar component (Figure 2b). The perichromatin granules were not labelled and IG clusters were almost devoid of gold grains (Figure $2 \mathrm{a}$ and $2 \mathrm{c}$ ). The ground cytoplasm generally contained diffuse labelling, although many gold particles were observed over free as well as membranebound ribosomes (Figure 2a). As for the nuclear bodies typical of hibernation, in deeply hibernating animals as well as in most liver samples submitted to in vitro conditions the $A B s$ were strongly labelled (Figure $2 \mathrm{C}$ ), whereas the CBs (Figure $2 \mathrm{~d}$ ) and the 
Table 1. Mean values $\pm S E$ of anti-CLOCK labeling densities (gold grains $/ \mu \mathrm{m}^{2}$ ) over various cellular compartments in euthermic and hibernating dormice. In each column values identified by asterisks are not significantly different from one another.

\begin{tabular}{lccccccc}
\hline \hline & CYTOPLASM & $\begin{array}{c}\text { CONDENSED } \\
\text { CHROMATIN }\end{array}$ & $\begin{array}{c}\text { INTERCHROMATIN } \\
\text { SPACE - IG }\end{array}$ & IG & NUCLEOLUS & ABS & CBS \\
\hline Euthermia & $4.51 \pm 0.58^{*}$ & $4.74 \pm 0.38^{*}$ & $8.12 \pm 0.61^{*}$ & $0.87 \pm 0.27$ & $6.48 \pm 2.04^{*}$ & - & - \\
Deep hibernation & $2.25 \pm 0.19$ & $1.68 \pm 0.21$ & $4.00 \pm 0.24$ & $0.43 \pm 0.14$ & $4.48 \pm 1.48$ & $85.70 \pm 6.88$ & $0.00 \pm 0.00$ \\
Early hibernation & $3.79 \pm 0.83^{*}$ & $4.17 \pm 0.48^{*}$ & $7.45 \pm 0.45^{*}$ & $0.51 \pm 0.15$ & $7.68 \pm 3.14^{*}$ & - & - \\
& & & & & & & - \\
\hline
\end{tabular}

Table 2. Mean values $\pm S E$ of anti-CLOCK labeling densities (gold grains $/ \mu \mathrm{m}^{2}$ ) over various cellular compartments in different in vitro experimental conditions. In each column values identified by asterisks are not significantly different from one another.

\begin{tabular}{|c|c|c|c|c|c|c|c|c|}
\hline & CYTOPLASM & $\begin{array}{l}\text { CONDENSED } \\
\text { CHROMATIN }\end{array}$ & $\begin{array}{l}\text { INTERCHROMATIN } \\
\text { SPACE- IG }\end{array}$ & $I G$ & NUCLEOLUS & ABs & CBs & FGM \\
\hline $4^{\circ} \mathrm{C} 5 \mathrm{~min}$ & $4.40 \pm 0.71^{*}$ & $2.14 \pm 0.50 *$ & $8.02 \pm 2.01 *$ & $0.00 \pm 0.00 *$ & $4.83 \pm 0.98 *$ & $73.13 \pm 13.89 *$ & $0.00 \pm 0.00 *$ & $0.21 \pm 0.10^{*}$ \\
\hline $4^{\circ} \mathrm{C} 30 \mathrm{~min}$ & $5.01 \pm 0.58 *$ & $1.83 \pm 0.36^{*}$ & $7.39 \pm 1.52 *$ & $0.11 \pm 0.02 *$ & $4.58 \pm 1.06^{*}$ & $85.91 \pm 17.61 *$ & $0.00 \pm 0.00 *$ & $0.00 \pm 0.00 *$ \\
\hline $4^{\circ} \mathrm{C} 60 \mathrm{~min}$ & $4.96 \pm 0.97 *$ & $2.09 \pm 0.41 *$ & $6.97 \pm 0.41 *$ & $0.70 \pm 0.70^{*}$ & $4.34 \pm 1.84^{*}$ & $16.77 \pm 16.77$ & not found & $0.11 \pm 0.11 *$ \\
\hline $4^{\circ} \mathrm{C} 24 \mathrm{~h}$ & $4.90 \pm 0.78 *$ & $2.12 \pm 0.51 *$ & $7.32 \pm 0.58 *$ & $0.78 \pm 0.12 *$ & $4.92 \pm 1.05^{*}$ & not found & not found & not found \\
\hline $37^{\circ} \mathrm{C} 5 \mathrm{~min}$ & $4.99 \pm 0.33 *$ & $1.49 \pm 0.57$ & $6.91 \pm 0.82 *$ & $0.43 \pm 0.17^{*}$ & $6.93 \pm 2.74$ & not found & not found & not found \\
\hline DADLE 5 min & $5.15 \pm 0.98^{*}$ & $2.17 \pm 0.66^{*}$ & $7.31 \pm 0.88^{*}$ & $0.35 \pm 0.11 *$ & $5.05 \pm 2.29 *$ & $97.31 \pm 17.65 *$ & $0.00 \pm 0.00 *$ & not found \\
\hline DADLE 30 min & $4.43 \pm 0.73^{*}$ & $1.45 \pm 0.31 *$ & $6.35 \pm 0.87^{*}$ & $0.77 \pm 0.56^{*}$ & $5.16 \pm 2.02 *$ & $0.00 \pm 0.00$ & $0.00 \pm 0.00 *$ & not found \\
\hline DADLE $60 \mathrm{~min}$ & $4.35 \pm 0.91 *$ & $2.03 \pm 0.48 *$ & $7.95 \pm 0.91 *$ & $0.93 \pm 0.13^{*}$ & $5.00 \pm 0.65 *$ & not found & not found & not found \\
\hline
\end{tabular}

FGM (Figure 2e) appeared devoid of gold grains. On the other hand, in liver samples incubated with DADLE for 30 and $60 \mathrm{~min}$ and at $4^{\circ} \mathrm{C}$ for $60 \mathrm{~min}$ and $24 \mathrm{~h}$ the rare ABs observed were either weakly labelled or unlabelled (not shown).

\section{Quantitative evaluation of immunolabelling and statistical analysis}

Quantitative evaluation of intracellular distribution of CLOCK protein confirmed that in all samples the most labelled compartments were the interchromatin space devoid of IG and the nucleolus, that in euthermia showed lower values. The condensed chromatin and the cytoplasm displayed similar densities with lower values in comparison to the nucleolus. The IG showed labelling densities not significantly different from background. Significant differences in labelling density were found between euthermic and deeply hibernating dormice (Table 1). In fact, in deep hibernation the anti-CLOCK labelling was significantly lower in the cytoplasm as well as in most nuclear compartments. By contrast, a striking accumulation of CLOCK protein was found in $A B s$, while $C B$ and $F G M$ exhibited values not significantly different from background. In the early hibernating animal all values were similar to the euthermic ones. In all liver samples submitted to in vitro conditions (Table 2) CLOCK labelling levels of cytoplasm and interchromatin space devoid of IG always showed euthermic values, whereas condensed chromatin and nucleolus maintained the hibernating values, with the exception of samples incubated at $37^{\circ} \mathrm{C}$ for 5 min. IG always displayed signals similar to background.

Quantitative evaluation of background labelling demonstrated that it was negligible $(0.15 \pm 0.04$ grains $/ \mu \mathrm{m}^{2}$ ). Similarly, the level of labelling in the control specimens was also negligible.

\section{Discussion}

Our observations, carried out on hepatocytes of euthermic and hibernating edible dormice, demonstrate that: 1) CLOCK protein is expressed in all 
animals and is more abundant in deep hibernation; 2 ) in all animals CLOCK protein mainly occurs in the nucleus and, in particular, on PF and nucleoli; 3) during deep hibernation CLOCK protein significantly decreases in all cellular compartments, but it accumulates in the ABs; 4 ) in hepatocytes submitted to in vitro conditions mimicking arousal CLOCK protein levels rapidly reach the euthermic values in the cytoplasm and in PF.

Clock gene encodes a bHLH-PAS transcription factor able to enhance transcription through $\mathrm{E}$ box elements (Reppert and Weaver, 2001) and is expressed in several tissues, including the liver (King et al., 1997). Accordingly, CLOCK protein has been specifically recognised in Glis glis liver homogenates, indicating a high homology between mouse and edible dormouse proteins. Moreover, the immunocytochemical analysis demonstrated that most CLOCK protein in dormouse hepatocytes is located in the nucleus and in particular on PF, nucleoplasmic structural constituents containing several transcription and splicing factors and representing the in situ form of nascent pre-mRNA (Fakan, 1994), and in the nucleolar dense fibrillar component, where the transcription and early splicing of rRNA take place (Cmarko et al., 2000; Biggiogera et al., 2001). As for the anti-CLOCK labelling observed on the condensed chromatin, it can not be excluded that some protein binds nontranscribing DNA; however, the absence of a clearcut borderline between the condensed chromatin and the perichromatin region could lead one to include the hardly recognisable PF in the chromatin area. On the other hand, CLOCK protein is absent from perichromatin granules, which represent storage and/or transport sites of already spliced mRNA or pre-mRNA (Vazquez-Nin et al., 1990; Bauren and Wieslander, 1994) and from the IG, involved in presplicing complex storage and/or assembly (Puvion and Puvion Dutilleul, 1996). Finally, the CLOCK protein found in the cytoplasm probably represents synthesised and migrating molecules.

Our observations are in agreement with a recent immunofluorescence study reporting a speckled distribution of CLOCK protein in the cytoplasm and in the nucleus (Zanello et al., 2000).

By comparing euthermic and hibernating animals it is evident that during deep hibernation liver CLOCK protein content increases but a concomitant intracellular redistribution takes place. More precisely, CLOCK protein decreases both in the cytoplasm and in the usual nuclear constituents, in accordance with the drastic reduction - but not the arrest - in protein and RNA synthesis occurring during hibernation (Derij and Shtark, 1985; Bocharova et al., 1992); however, at the same time large amounts of this protein are stored in ABs. These particular nuclear bodies progressively form during the hibernation bouts becoming quite abundant in deeply hibernating dormice (about 15\% of sectioned hepatocyte nuclei show at least one $A B$, and we could observe up to three $A B s$ per nuclear section) (Malatesta et al., 1999). ABs have been demonstrated to contain snRNPs and hnRNPs (Malatesta et al., 1994, 1999) and are thought to represent storage and/or assembly sites of such molecules requested to meet the increased metabolic needs upon arousal, when they disassemble in thin fibrils (Malatesta et al., 2001). Similarly, we can hypothesise that $A B s$ represent storage sites of CLOCK protein to be rapidly used upon arousal in order to start a massive transcription process. In fact, our in vitro investigations demonstrate a striking increase in CLOCK protein levels on PF after an incubation of a few minutes in all experimental conditions, whereas the rare ABs found in liver samples incubated for longer times are almost devoid of CLOCK protein. On the other hand, CLOCK protein is absent from CBs, which are supposed to play a role in the metabolism of many nucleoplasmic and nucleolar transcription and splicing factors, and which perhaps shuttle such proteins to and from different nucleoplasmic/nucleolar sites (Malatesta et al., 1994; 1999). However, some factors, such as SC-35, are absent from CBs. Similarly, CLOCK protein could follow intranuclear pathways not involving CBs. CLOCK protein is also absent from the FGM, probably involved in post-transcriptional processing of pre-mRNA slowly formed during hibernation (Malatesta et al., 1995, 1999).

The literature data on the circadian fluctuations during hibernation are quite poor and appear to differ among species (Körtner and Geiser, 2000). It has been reported that hibernators which use deep and secluded underground burrows, show no surface activity and rely entirely on body fat stores or food caches, such as Glis glis, are characterised by a deterioration of the circadian system during the hibernation bouts. This would be in accordance with the decrease of CLOCK protein in both synthetic and functional sites. Moreover, it is known that hibernators arouse from torpor at regular intervals, 
whose biological significance is still unclear (Lyman et al., 1982). Some authors propose that such periodic normothermic episodes would reestablish homeostatic balance, others that they would reflect the persistence of a lengthened circadian cycle (Körtner and Geiser, 2000). In any case, our data suggest that the periodic arousals could reset the cellular circadian clock: upon arousal CLOCK protein synthesis and distribution on functional sites would be quickly restored (as indicated by our in vitro experiments), while during each hibernating bout the protein would be progressively concentrated in the ABs (as indicated by the early hibernating dormouse, showing CLOCK protein content and distribution quite similar to the euthermic ones). Interestingly, dormouse hepatic tissue appears to be able to restore the intracellular circadian clock even under in vitro condition, in the absence of inputs from the hypothalamic master oscillator. It is possible that stimuli such as temperature changes (Liu et al., 1998) or humoral signals (Balsalobre et al., 1998) can trigger the intracellular timekeeping system, whereas it has been excluded that mammalian peripheral clocks can respond directly to light (Yamazaki et al., 2000).

\section{References}

Balsalobre A, Damiola, F, Schibler U: A serum shock induces circadian gene expression in mammalian tissue culture cells. Cell, 93, 929937, 1998.

Bauren $G$, Wieslander L: Splicing of Balbiani ring-1 gene pre messenger RNA occurs simultaneously with transcription. Cell, 76, 183192, 1994.

Bernhard, W: A new staining procedure for electron microscopical cytology. J. Ultrastruct. Res, 27, 250-265, 1969.

Biggiogera, M, Malatesta, M, Abolhassani-Dadras, S, Amalric, F Rothblum, L.I, and Fakan, S: Revealing the unseen: the organizer region of the nucleolus. J. Cell Sci, 114, 3199-3205, 2001.

Bocharova, L.S, Gordon, R.Y, and Arkhipov, V.I: Uridine uptake and RNA synthesis in the brain of torpid and awakened ground squirrels. Comp. Biochem. Physiol, 101B, 189-192, 1992.

Bradford, M.M: A rapid and sensitive method for the quantitation of microgram quantities of protein utilizing the principle of protein-dye binding. Anal. Biochem, 72, 248-254, 1976.

Cmarko, D, Verschure, P.J, Rothblum, L.I, Hernandez-Verdun, D, Amalric, F, van Driel, R, and Fakan, S: Ultrastructural analysis of nucleolar transcription in cells microinjected with 5-bromo-UTP. Histochem. Cell Biol, 113, 181-187, 2000.

Derij, L.V, and Shtark, M.B: Hibernators' brain: protein synthesis in the neocortex and the hippocampus. Comp. Biochem. Physiol, 80B, 927-934, 1985.

Fakan, S: Perichromatin fibrils are in situ forms of nascent transcripts. Trends Cell Biol, 4, 86-90, 1994.

King, D.P, Zhao, Y, Sangoram, A.M, Wilsbacher, L.D, Tanaka, M, Antoch, M.P, Steeves, T.D.L, Vitaterna, M.H, Kornhauser, J.M Lowrey, P.L, Turek, F.W, and Takahashi, J.S: Positional cloning of the mouse circadian Clock gene. Cell, 89, 641-653, 1997.
Körtner, G, and Geiser, F: The temporal organization of daily torpor and hibernation: ciecadian and circannual rhythms. Chronobiol. Int, 17, 103-128, 2000.

Laemmli, U.K: Cleavage of structural proteins during the assembly of the head of bacteriophage. Nature, 227, 680-685, 1970.

Liu, Y, Merrow, M, Loros, J.J, and Dunlap, J.C: How temperature changes reset a circadian oscillator. Science, 281, 825-829, 1998.

Lyman, C.P, Willis, J.S, Malan, A, and Wang, L.C.H: Hibernation and torpor in mammals and birds. Academic Press, New York, 1982.

Malatesta, M, Gazzanelli, G, Battistelli, S, Martin, T.E, Amalric, F, and Fakan, S: Nucleoli undergo structural and molecular modifications during hibernation. Chromosoma, 109, 506-513, 2000.

Malatesta, M, Luchetti, F, Marcheggiani, F, Fakan, S, and Gazzanelli, $G$ : Disassembly of nuclear bodies during arousal from hibernation: an in vitro study. Chromosoma, 110, 471-477, 2001.

Malatesta, M, Zancanaro, C, Martin, T.E, Chan, E.K.L, Almaric, F, Luhrmann, R, Vogel, P, and Fakan, S: Cytochemical and immunocytochemical characterization of nuclear bodies during hibernation. Eur. J. Cell Biol, 65, 82-93, 1994.

Malatesta, M, Zancanaro, C, Tamburini, M, Martin, T.E, Fu, X.-D, Vogel, $\mathrm{P}$, and Fakan, $\mathrm{S}$ : Novel nuclear ribonucleoprotein structural components in the dormouse adrenal cortex during hibernation. Chromosoma, 104, 121-128, 1995.

Malatesta, M, Cardinali, A, Battistelli, S, Zancanaro, C, Martin, T.E, Fakan, S, and Gazzanelli, G: Nuclear bodies are usual constituents in tissues of hibernating dormice. Anat. Rec, 254, 389-395, 1999.

Oeltgen, P.R, Nilekani, S.P, Nuchols, P.A, Spurrier, W.A, and Su, T.P: Further studies on opioids and hibernation: delta opioid receptor ligand selectively induced hibernation in summer-active ground squirrels. Life Sci, 43, 1565-1574, 1988.

Puvion, $E$, and Puvion-Dutilleul, F: Ultrastructure of the nucleus in relation to transcription and splicing: roles of perichromatin fibrils and interchromatin granules. Exp. Cell Res, 229, 217-225, 1996.

Reppert, S.M, and Weaver, D.R: Molecular analysis of mammalian circadian rhythms. Annu. Rev. Physiol, 63, 647-676, 2001.

Sakamoto, K, Nagase, T, Fukui, H, Horikawa, K, Okada, T, Tanaka, H, Sato, K, Miyake, Y, Ohara, O, Kako, K, and Ishida, N: Multitissue circadian expression of rat period homolog ( $r$ Per2) mRNA is governed by the mammalian circadian clock, the suprachiasmatic nucleus in the brain. J. Biol. Chem, 273, 27039-27042, 1998.

Towbin, H, Staehelin, T. and Gordon, J: Electrophoretic transfer of proteins from polyacrylamide gels to nitrocellulose sheets: procedure and some applications. Proc. Natl Acad. Sci. USA, 76, 4350-4354, 1979.

Vazquez-Nin, G.H, Echeverria, O.M, Fakan, S, Leser, G, and Martin, T.E: Immunoelectron microscope localization of snRNPs in the polytene nucleus of salivary glands of Chironomus thummi. Chromosoma, 99, 44-51, 1990.

Vitaterna, M.H, King, D.P, Chang, A.M, Kornhauser, J.M, Lowrey, P.L, McDonald, J.D, Dove, W.F, Pinto, L.H, Turek, F.W, and Takahashi, J.S: Mutagenesis and mapping of a mouse gene, Clock, essential for circadian behaviour. Science, 264, 719-725, 1994.

Whitmore, D, Foulkes, N.S, Strähle, U, and Sassone-Corsi, P: Zebrafish Clock rhythmic expression reveals independent peripheral circadian oscillators. Nature Neuroscience, 1, 701-707, 1998.

Yamazaki, S, Numno, R, Abe, M, Hida, A, Takahashi, R, Ueda, M, Block, G.D, Sakaki, Y, Menaker, M, and Tei, H: Resetting central and peripheral circadian oscillators in transgenic mice. Science, 288, 682-685, 2000.

Zancanaro, C, Malatesta, M, Vogel, P, Osculati, F, and Fakan, S: Ultrastructural and morphometrical analyses of the brown adipocyte nucleus in a hibernating dormouse. Biol. Cell, 79, 55-61, 1993.

Zanello, S.B, Jackson, D.M, and Holick, M.F: Expression of the circadian clock genes clock and periodl in human skin. J. Invest. Dermatol, 115, 757-760, 2000.

Zylka, M.J, Shearnan, L.P, Weaver, D.R, and Reppert, S.M: Three period homologs in mammals: differential light responses in the suprachiasmatic circadian clock and oscillating transcripts outside of brain. Neuron, 20, 1-20, 1998. 